\title{
A Parallel Finite Element Algorithm for the Unsteady Oseen Equations
}

\author{
Qi Ding ${ }^{1,2}$ and Yueqiang Shang ${ }^{1, *}$ \\ ${ }^{1}$ School of Mathematics and Statistics, Southwest University, Chongqing 400715, China \\ 2 Tongnan Middle School, Chongqing 402660, China
}

Received 12 September 2019; Accepted (in revised version) 15 December 2020

\begin{abstract}
Based on fully overlapping domain decomposition, a parallel finite element algorithm for the unsteady Oseen equations is proposed and analyzed. In this algorithm, each processor independently computes a finite element approximate solution in its own subdomain by using a locally refined multiscale mesh at each time step, where conforming finite element pairs are used for the spatial discretizations and backward Euler scheme is used for the temporal discretizations, respectively. Each subproblem is defined in the entire domain with vast majority of the degrees of freedom associated with the particular subdomain that it is responsible for and hence can be solved in parallel with other subproblems using an existing sequential solver without extensive recoding. The algorithm is easy to implement and has low communication cost. Error bounds of the parallel finite element approximate solutions are estimated. Numerical experiments are also given to demonstrate the effectiveness of the algorithm.
\end{abstract}

AMS subject classifications: 68W10, 65M15, 76M10, 76D05

Key words: Oseen equations, finite element, overlapping domain decomposition, backward Euler scheme, parallel algorithm.

\section{Introduction}

In the last decades, based on the idea of $\mathrm{Xu}$ and Zhou [1-3] for local and parallel finite element discretizations, some new local and parallel algorithms have been proposed for eigenvalue problems [3-5], the stationary incompressible magnetohydro dynamicsthe [6], the steady Stokes equations [7-10], the Stokes/Darcy problem [11], the steady Navier-Stokes equations [12-25] and the stream function form of Navier-Stokes equations [26]. These algorithms have less communication complexity than current standard approaches and allow existing sequential PDE codes to run in a parallel environment

*Corresponding author.

Emails: 17783023729@163.com (Q. Ding),yqshang@swu.edu.cn (Y. Q. Shang) 
without a large investment in recoding. It is shown by numerical experiments and comparing results that these algorithms are highly efficient and easy to implement.

Our ultimate goal is to study local and parallel finite element discretization algorithms for the unsteady incompressible Navier-Stokes equations. By linearizing the incompressible Navier-Stokes equations (e.g., by a semi-implicit iteration), we get an Oseen problem, which shows up as an auxiliary problem in many numerical approaches for solving the Navier-Stokes equations. Therefore, it is reasonable to study the Oseen problem first. For the Oseen problem, some numercial methods were proposed and analyzed such as the weak Galerkin finite element methods [27], the Nitsche cut finite element methods [28], the stabilized Nitsche cut finite element methods [29] and the new streamline diffusion finite element methods [30]. However, to the authors' best knowledge, there is no study on local and parallel finite element discretizations for the unsteady Oseen problems.

We notice that local and parallel finite element algorithms for the unsteady Stokes and Navier-Stokes equations were investigated numerically in [31] and [32], respectively. However, there is a lack of theoretical analysis in the above works. In this paper, we extend our work for the unsteady Stokes equations in [33] to the unsteady Oseen problem. Compared with the Stokes equations, the Oseen equations possess a convective term $b \cdot \nabla u$, which has a significant impact on the theoretical analysis and numerical computation. With backward Euler scheme for the temporal discretizations, we develop a parallel fully discrete finite element algorithm, in which each processor independently computes a finite element approximation solution in its own subdomain by using locally refined multiscale mesh at each time step. Meanwhile, using the theoretical tool of local a priori error estimate for the finite element discretization solutions, error bounds of the obtained finite element approximations from our parallel algorithm are derived.

The outline of the paper is as follows. In the next section, some mathematical preliminaries and assumptions on the mixed finite element spaces are given. In Section 3, local finite element method is given and error estimates are derived, which play an important role in analysing the proposed algorithms in the next section. In Section 4, based on fully overlapping domain decomposition technique, a parallel finite element method for solving the unsteady Oseen equation is proposed. Numerical experiments are given to verify the effectiveness of the algorithm in Section 5. Finally, some conclusions in Section 6 are obtained.

\section{Mathematical preliminaries}

Let $\Omega$ be a bounded domain with Lipschitz-continuous boundary $\partial \Omega$ in $\mathbb{R}^{2}$ and $(0, T)$ be a time interval with $T<\infty$. we shall use the standard notations $W^{m, p}(\Omega)$ for Sobolev spaces and their associated norms $\|\cdot\|_{m, p}$ and seminorms $|\cdot|_{m, p}$ (cf. [34-36]). For $p=2$, we denote $H^{m}(\Omega)=W^{m, 2}(\Omega)$ and then denote by $H_{0}^{1}(\Omega)$ the closed subspace of $H^{1}(\Omega)$ consisting of functions with zero trace on $\partial \Omega$. The space $H^{-1}(\Omega)$, the dual of $H_{0}^{1}(\Omega)$ and its associated 
norm $\|\cdot\|_{-1, \Omega}$ will also be used. Moreover, for any Banach space $X, L^{p}(0, T ; X)$ denotes the space of measurable $X$-valued functions $f=f(t)$ with $t \in(0, T)$, such that

$$
\|f\|_{L^{p}(0, T ; X)}=\left(\int_{0}^{T}\|f(t)\|_{X}^{p} d t\right)^{1 / p}<\infty,
$$

if $1 \leq p<\infty$, or such that

$$
\|f\|_{L^{\infty}(0, T ; X)}=\operatorname{ess} \sup _{0<t \leq T}\|f(t)\|_{X}<\infty,
$$

if $p=\infty$.

For a subdomain $\Omega_{0} \subset \Omega$, we view $H_{0}^{1}\left(\Omega_{0}\right)$ as a subspaces of $H_{0}^{1}(\Omega)$ by extending the functions in $H_{0}^{1}\left(\Omega_{0}\right)$ to be functions in $H_{0}^{1}(\Omega)$ with zero outside of $\Omega_{0}$. Throughout this paper, we shall use the letter $\mathrm{c}$ (with or without subscripts) to denote a generic positive constant which is independent of the mesh parameter and time step size and may stand for different values at its different occurrences.

\subsection{The Oseen equations}

We consider the following unsteady Oseen equations:

$$
\begin{array}{ll}
u_{t}-v \Delta u+(b \cdot \nabla) u+\nabla p=f & \text { in } \Omega \times(0, T], \\
\nabla \cdot u=0 & \text { in } \Omega \times(0, T], \\
u=0 & \text { on } \partial \Omega \times(0, T], \\
u=u_{0} & \text { on } \Omega \times\{0\},
\end{array}
$$

where $u:(0, T) \times \Omega \rightarrow R^{2}$ represents the velocity vector, $p:(0, T) \times \Omega \rightarrow R$ the pressure, $f:(0, T) \times \Omega \rightarrow R^{2}$ the external body force, $v>0$ the kinematic viscosity, $u_{0}: \Omega \rightarrow R^{2}$ the initial velocity satisfying

$$
\nabla \cdot u_{0}=0, \quad u_{t}=\frac{\partial u}{\partial t}
$$

and $b:(0, T) \times \Omega \rightarrow R^{2}$ a solenoidal vector field satisfying $\nabla \cdot b=0$.

To introduce the weak form of Eqs. (2.1a)-(2.1d), we set

$$
X=H_{0}^{1}(\Omega)^{2}, \quad Y=L^{2}(\Omega)^{2}, \quad M=L_{0}^{2}(\Omega)=\left\{q \in L^{2}(\Omega): \int_{\Omega} q d x=0\right\} .
$$

Let the closed subset $V$ of $X$ be given by

$$
V=\{v \in X, \nabla \cdot v=0\} .
$$

We denote by $V_{1}$ the closed subset of $Y$,

$$
V_{1}=\left\{v \in Y, \nabla \cdot v=0,\left.v \cdot n\right|_{\partial \Omega}=0\right\} .
$$


Besides, we need some assumptions on the solution domain, the prescribed data and the true solutions as follows (cf. [37-39]).

(A1). The unique solution $(v, q) \in X \times M$ of the steady Stokes problem

$$
-v \Delta v+\nabla q=g, \quad \nabla \cdot v=0 \quad \text { in } \Omega,\left.\quad v\right|_{\partial \Omega}=0,
$$

for prescribed $g \in H^{k-2}$, for any $2 \leq k \leq 3$, satisfies

$$
\|v\|_{k, \Omega}+\|q\|_{k-1, \Omega} \leq c\|g\|_{k-2, \Omega} .
$$

(A2). The initial velocity $u_{0} \in V \cap H^{2}(\Omega)^{2}$, the solenoidal vector field $b \in L^{\infty}\left(0, T ; W^{1, \infty}(\Omega)^{2}\right)$ and the prescribed force $f$ is smooth uniformly in time, satisfying

$$
\left\|u_{0}\right\|_{2, \Omega}+\sup _{t \in(0, T]}\left\{\left\|\partial_{t}^{k} f\right\|_{m, \Omega}+\|b\|_{1, \infty, \Omega}\right\} \leq c, \quad k, m=0,1,2 .
$$

(A3). There exists a constant $C$ such that

$$
\sup _{t \in(0, T]}\|\nabla u(\cdot, t)\|_{0, \Omega} \leq C .
$$

Given $f \in L^{\infty}(0, T ; Y)$, the weak form of (2.1a)-(2.1d) reads: find a pair of $(u, p) \in X \times M$ for $0<t \leq T$ such that

$$
\begin{aligned}
& \left(u_{t}, v\right)+B((u, p) ;(v, q))+N(b, u, v)=(f, v), \quad \forall(v, q) \in X \times M, \\
& u(0)=u_{0},
\end{aligned}
$$

where $(\cdot, \cdot)$ denotes the standard inner-product of $L^{2}(\Omega)$ or $L^{2}(\Omega)^{2}$,

$$
\begin{aligned}
& B((u, p) ;(v, q))=a(u, v)-d(v, p)+d(u, q), \\
& a(u, v)=v(\nabla u, \nabla v), \quad d(v, q)=(\nabla \cdot v, q), \quad \forall u, v \in X, q \in M,
\end{aligned}
$$

and $N(\cdot, \cdot, \cdot)$ is defined as

$$
\begin{aligned}
N(u, v, w) & =((u \cdot \nabla) v, w)+\frac{1}{2}((\nabla \cdot u) v, w) \\
& =\frac{1}{2}((u \cdot \nabla) v, w)-\frac{1}{2}((u \cdot \nabla) w, v), \quad \forall u, v, w \in X,
\end{aligned}
$$

which has the following properties (see [34,39]):

$$
\begin{aligned}
& N(u, v, w)=-N(u, w, v), \quad \forall u, v, w \in X \text {, } \\
& |N(b, v, w)| \leq\|b\|_{L^{\infty}}\|\nabla v\|_{0, \Omega}\|w\|_{0, \Omega}, \quad \forall v, w \in X .
\end{aligned}
$$

For the existence, uniqueness and regularity of a solution of problem (2.2), we have the following results. 
Theorem 2.1. Assume that assumptions (A1), (A2) and (A3) hold. Then problem (2.2) admits a unique solution pair $(u, p) \in\left(L^{2}\left(0, T ; H^{2}(\Omega)^{2}\right) \cap C(0, T ; V) \cap H^{1}\left(0, T ; L^{2}(\Omega)^{2}\right) \times L^{2}\left(0, T ; H^{1}(\Omega) \cap\right.\right.$ $M)$. Moreover, the following bounds hold:

$$
\begin{aligned}
& \sup _{0<t \leq T}\left(\left\|u_{t}(t)\right\|_{k-2, \Omega}^{2}+\|u(t)\|_{k, \Omega}^{2}+\|p(t)\|_{k-1, \Omega}^{2}\right) \leq c, \quad k=2,3 \\
& \sup _{0<t \leq T}\left\|u_{t}(t)\right\|_{1, \Omega}^{2}+\int_{0}^{T}\left(\left\|u_{t t}(t)\right\|_{0, \Omega}^{2}+\left\|u_{t}(t)\right\|_{2, \Omega}^{2}+\left\|p_{t}(t)\right\|_{1, \Omega}^{2}\right) d t \leq c .
\end{aligned}
$$

Remark 2.1. Noting that the Oseen problem can be viewed as a linear model of the Navier-Stokes equations as mention in Introduction section and then regularities of a solution of the Oseen problem can be obtained from those of the Navier-Stokes equations by a slight modification to the corresponding arguments (i.e., by changing the nonlinear term of the unsteady Navier-Stokes equations to the linear term of the unsteady Oseen equations). For the Navier-Stokes equations, the existence and uniqueness of the solution are referred to Theorem 2.1 in Hill and Sli [37], while for (2.4a)-(2.4b), we refer to Theorems 2.3-2.5 in Heywood and Rannacher [39] and Theorem 2.2 in Hill and Sli [37].

\subsection{Mixed finite element spaces}

Let $0<h<1$ be a real positive parameter and $T^{h}(\Omega)=\{K\}$ be a regular triangulation of $\bar{\Omega}$ into triangles or quadrilaterals $K$ with mesh size $h$. The mesh $T^{h}(\Omega)$ is assumed to be uniformly regular as $h \rightarrow 0$ (see [1] for details). Let $X_{h}(\Omega) \subset H^{1}(\Omega)^{2}, M_{h}(\Omega) \subset L^{2}(\Omega)$ be two finite element subspaces associated with the mesh $T^{h}(\Omega)$ and

$$
X_{h}^{0}(\Omega)=X_{h}(\Omega) \cap X, \quad M_{h}^{0}(\Omega)=M_{h}(\Omega) \cap M .
$$

Given $G \subset \subset \Omega_{0} \subset \Omega$ (here and hereafter, the notation $G \subset \subset \Omega_{0}$ means that $\operatorname{dist}\left(\partial G \backslash \partial \Omega, \partial \Omega_{0} \backslash \partial \Omega\right)>0$; see Fig. 1), we define $T^{h}(G), X_{h}(G)$ and $M_{h}(G)$ to be the restriction of $T^{h}(\Omega), X_{h}(\Omega)$ and $M_{h}(\Omega)$ to $G$, respectively and

$$
X_{0}^{h}(G)=\left\{v \in X_{h}(\Omega): \operatorname{supp} v \subset \subset G\right\}, \quad M_{0}^{h}(G)=\left\{q \in M_{h}(\Omega): \operatorname{supp} q \subset \subset G\right\} .
$$

The assumptions on the mixed finite element spaces are as follows (cf. [1-3, 13,40-43]):

(B1). Approximation. For each $(u, p) \in\left(H^{3}(G)^{2} \cap X\right) \times\left(H^{2}(G) \cap M\right)$, there exists an approximation $\left(\pi_{h} u, \rho_{h} p\right) \in X_{h}(G) \times M_{h}(G)$ such that

$$
\begin{array}{ll}
\left\|h^{-1}\left(u-\pi_{h} u\right)\right\|_{0, G}+\left\|u-\pi_{h} u\right\|_{1, G} \leq c h^{s}\|u\|_{1+s, G}, & s=1,2, \\
\left\|h^{-1}\left(p-\rho_{h} p\right)\right\|_{-1, G}+\left\|p-\rho_{h} p\right\|_{0, G} \leq c h^{s}\|p\|_{s, G}, & s=1,2 .
\end{array}
$$

(B2). Inverse estimate. For any $(v, q) \in X_{h}(G) \times M_{h}(G)$, there hold

$$
\|v\|_{1, G} \leq c\left\|h^{-1} v\right\|_{0, G}, \quad\|q\|_{0, G} \leq c\left\|h^{-1} q\right\|_{-1, G} .
$$



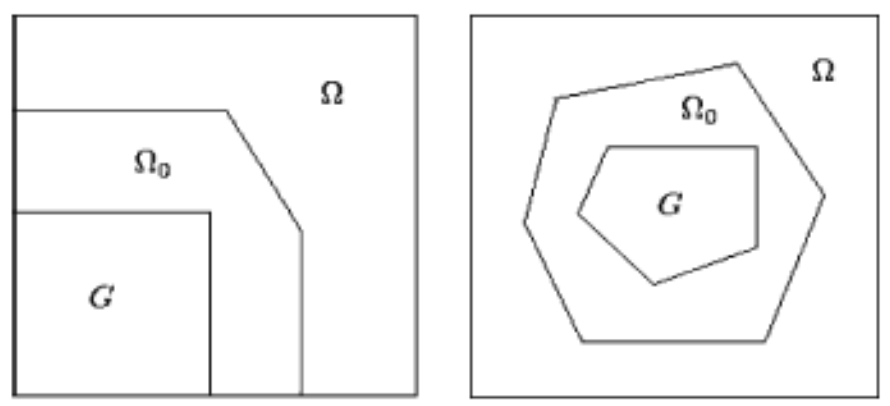

Figure 1: Subdomains $G \subset \Omega_{0} \subset \Omega$ with $G \subset \subset \Omega_{0} \subset \Omega$.

(B3). Superapproximation. For $G \subset \Omega_{0}$, let $\omega \in C_{0}^{\infty}(\Omega)$, with supp $\omega \subset \subset G$. Then for any $(u, p) \in X_{h}(G) \times M_{h}(G)$, there is $(v, q) \in X_{0}^{h}(G) \times M_{0}^{h}(G)$ such that

$$
\left\|h^{-1}(\omega u-v)\right\|_{1, G} \leq c\|u\|_{1, G}, \quad\left\|h^{-1}(\omega p-q)\right\|_{0, G} \leq c\|p\|_{0, G} .
$$

(B4). Stability. There exists a constant $\beta>0$ such that

$$
\beta\|q\|_{0, G} \leq \sup _{v \in X_{h}^{0}(G), v \neq 0} \frac{(\nabla \cdot v, q)}{\|\nabla v\|_{0, G}}, \quad \forall q \in M_{h}^{0}(G) .
$$

The approximation property (B1) is referred to [41] and the inverse estimate property (B2) is referred to [1,2]. Many finite element spaces are known to have the superapproximation property (B3) (cf. [42-44]). When $G=\Omega$, the stability condition (B4) is standard for the mixed finite element spaces for the Oseen or Navier-Stokes equations and the readers are referred to [45] for a detailed proof. Many finite element spaces are known to satisfy the above mentioned Assumptions (B1)-(B4). The readers are referred to $[3,7,12,30,40]$ for some of these finite element spaces. For instance, the MINI finite elements [46] and the $P_{2}-P_{0}$ finite elements [47] satisfy Assumptions (B1)-(B4) when $k=1$, while the Taylor-Hood elements [48] and the augmented $P_{2}-P_{1}$ elements $[49,50]$ satisfy Assumptions (B1)-(B4) when $k=2$. Throughout this paper, (A1)-(A3) and (B1)-(B4) are assumed to be satisfied.

Under above assumptions, the mixed finite element approximations of problem (2.2) reads: find a pair of $\left(u^{h}, p^{h}\right) \in X_{h}^{0}(\Omega) \times M_{h}^{0}(\Omega)$ for $0<t \leq T$ such that

$$
\begin{aligned}
& \left(u_{t}^{h}, v\right)+B\left(\left(u^{h}, p^{h}\right) ;(v, q)\right)+N\left(b, u^{h}, v\right)=(f, v), \quad \forall(v, q) \in X_{h}^{0}(\Omega) \times M_{h}^{0}(\Omega), \\
& u^{h}(0)=P_{h} u_{0},
\end{aligned}
$$

where $P_{h}: Y \rightarrow X_{h}(\Omega)$ is the $L^{2}$-orthogonal projection defined by

$$
\left(P_{h} \omega, v^{h}\right)=\left(\omega, v^{h}\right), \quad \forall \omega \in Y, \quad v^{h} \in X_{h}(\Omega) .
$$


Theorem 2.2. Under the conditions of Theorem 2.1 and assume (B1), (B2) and (B4) hold. Then problem (2.5) has a unique solution pair $\left(u^{h}, p^{h}\right) \in L^{\infty}\left(0, T ; X_{h}^{0}(\Omega)\right) \times L^{\infty}\left(0, T ; M_{h}^{0}(\Omega)\right)$. Provided that $(u, p) \in L^{\infty}\left(0, T ; H^{3}(\Omega)^{2} \cap X\right) \times L^{2}\left(0, T ; H^{2}(\Omega) \cap M\right)$, then the following estimates hold for all $t \in(0, T]$

$$
\begin{array}{ll}
\left\|u(t)-u^{h}(t)\right\|_{0, \Omega}+h\left(\left|u(t)-u^{h}(t)\right|_{1, \Omega}+\left\|p(t)-p^{h}(t)\right\|_{0, \Omega}\right) \leq c h^{s+1}, & s=1,2, \\
\left\|u_{t}(t)-u_{t}^{h}(t)\right\|_{0, \Omega} \leq c h^{s+1}, & s=1,2 .
\end{array}
$$

Remark 2.2. The existence and uniqueness of the solution pair $\left(u^{h}, p^{h}\right) \in L^{\infty}\left(0, T ; X_{h}^{0}(\Omega)\right) \times$ $L^{\infty}\left(0, T ; M_{h}^{0}(\Omega)\right)$ of problem $(2.5)$ can be proved using a similar approach as described on page 495 in [38]. The error estimates are also can be obtained by similar arguments to those for the Navier-Stokes euqations.

\subsection{Fully discrete finite element scheme}

With backward Euler scheme for temporal discretization, the fully discrete scheme of problem (2.2) reads: find a pair $\left(u_{n}^{h}, p_{n}^{h}\right) \in X_{h}^{0}(\Omega) \times M_{h}^{0}(\Omega)$ such that

$$
\begin{aligned}
& \frac{1}{\tau}\left(u_{n}^{h}-u_{n-1}^{h}, v\right)+B\left(\left(u_{n}^{h}, p_{n}^{h}\right) ;(v, q)\right)+N\left(b, u_{n}^{h}, v\right) \\
& =\left(f_{n}, v\right), \quad \forall(v, q) \in X_{h}^{0}(\Omega) \times M_{h}^{0}(\Omega), \\
& u_{0}^{h}=u^{h}(0),
\end{aligned}
$$

where $\tau$ is the time-step size satisfying

$$
0<\tau<1, \quad f_{n}=\frac{1}{\tau} \int_{t_{n-1}}^{t_{n}} f d t, \quad t_{n}=n \tau, \quad n=1,2, \cdots, N, \quad \text { and } \quad N=[T / \tau] .
$$

For scheme (2.7), we have the following Theorems 2.3 and 2.4 with respective to the stability and error estimates.

Theorem 2.3. Under the conditions of Theorems 2.1 and 2.2, problem (2.7) admits a unique solution pair $\left(u_{n}^{h}, p_{n}^{h}\right) \in X_{h}^{0}(\Omega) \times M_{h}^{0}(\Omega)$ for $m=1,2, \cdots, N$, satisfying the following stability estimates

$$
\begin{aligned}
& \left\|u_{m}^{h}\right\|_{0, \Omega}^{2}+\sum_{n=1}^{m}\left\|u_{n}^{h}-u_{n-1}^{h}\right\|_{0, \Omega}^{2}+\sum_{n=1}^{m} v \tau\left|u_{n}^{h}\right|_{1, \Omega}^{2} \leq \frac{C_{\Omega}}{v}\|f\|_{L^{2}(0, T ; Y)}^{2}+\left\|u_{0}\right\|_{0, \Omega}^{2}, \\
& \tau \sum_{n=1}^{m}\left\|p_{n}^{h}\right\|_{0, \Omega}^{2} \leq \frac{c\left(1+C_{\Omega}^{2}\right)}{\beta^{2}}\left(\|f\|_{L^{2}(0, T ; Y)}^{2}+\left\|u_{0}\right\|_{0, \Omega}^{2}+\left|u_{0}\right|_{1, \Omega}^{2}\right),
\end{aligned}
$$

where $C_{\Omega}$ is the constant in the Poincaré inequality. 
Theorem 2.4. Let the conditions of Theorems 2.1 and 2.2 hold. Assume the following regularities for the solution $(u, p)$ of the Oseen equations (2.2) hold:

$$
u_{t}, u_{t t} \in L^{4}\left(0, T ; L^{2}(\Omega)^{2}\right), \quad u \in L^{4}\left(0, T ; W^{1, \infty}(\Omega)^{2}\right), \quad p \in L^{2}\left(0, T ; L^{2}(\Omega)\right) .
$$

Then the following error estimates are valid for all $n$

$$
\begin{array}{ll}
\left\|u\left(t_{n}\right)-u_{n}^{h}\right\|_{0, \Omega}+h\left|u\left(t_{n}\right)-u_{n}^{h}\right|_{1, \Omega} \leq c\left(\tau+h^{s+1}\right), & s=1,2, \\
\left\|p\left(t_{n}\right)-p_{n}^{h}\right\|_{0, \Omega} \leq c\left(\tau+h^{s}\right), & s=1,2 .
\end{array}
$$

Remark 2.3. The existence and uniqueness of a solution of (2.7) can be proved with LaxMilgram theorem; see the remarks on page 413 of [45]. The stability estimates (2.8a)-(2.8b) can be obtained by a standard argument like that for Lemma 7.77 in [45]. By similar arguments, the error estimates $(2.9 \mathrm{a})$ and $(2.9 \mathrm{~b})$ could be gotten by referring the proofs of Theorem 7.78 in [45] and Theorem 6.5 in [51] (where errors between the semi-discrete finite element solutions and the fully discrete finite element solutions are estimated, which combined with Theorem 2.2 can yield the required results).

\section{Local finite element algorithm}

In this section, we shall propose a local finite element algorithm for the unsteady Oseen equations and the local priori error estimate will be derived.

Assume $T^{H}(\Omega)$ be a global coarse grid with the mesh size $H \gg h, T^{h}\left(\Omega_{0}\right)$ a local fine grid with mesh size $h$, where $\Omega_{0}$ is a larger subdomain containing a subdomain $D$ (i.e., $\left.D \subset \subset \Omega_{0} \subset \Omega\right)$ and $T_{H}^{h}(\Omega)$ a composite mesh which is fine around the subdomian $D$ in such a way that $T_{H}^{h}\left(\Omega_{0}\right)=T^{h}\left(\Omega_{0}\right)$ and coarse far away from $D$ with size $H$. This composite mesh $T_{H}^{h}(\Omega)$ can be obtained by locally refining the coarse grid $T^{H}(\Omega)$ and then using some adaptive processes to make it compatibility and shape-regularity (a mesh or triangulation is said to be compatible if the intersection of two elements is either empty, a common vertex, a common side or a common face (see [35, 36, 41]). In our theoretical analysis, we need an auxiliary global grid $T^{h}(\Omega)$, which is assumed to coincide with $T_{H}^{h}(\Omega)$ on $\Omega_{0}$. Associated with the composite mesh $T_{H}^{h}(\Omega)$, we denote the corresponding finite element spaces by $X_{H, h}^{0}(\Omega) \subset X, M_{H, h}^{0}(\Omega) \subset M$ satisfying assumptions (A1)-(A4). In the following algorithm, we can obtain a local approximation solution in the subdomain $D$ by solving the Oseen equations on the global composite mesh $T_{H}^{h}(\Omega)$, where the subomain contain the vast majority of degrees of freedom of $T_{H}^{h}(\Omega)$.

Algorithm 3.1 (Local finite element algorithm). Find $\left(u_{n}^{H, h}, p_{n}^{H, h}\right) \in X_{H, h}^{0}(\Omega) \times M_{H, h}^{0}(\Omega)$, for $n=1,2, \cdots, N$, such that

$$
\begin{aligned}
& \frac{1}{\tau}\left(u_{n}^{H, h}-u_{n-1}^{H, h}, v\right)+B\left(\left(u_{n}^{H, h}, p_{n}^{H, h}\right) ;(v, q)\right)+N\left(b, u_{n}^{H, h}, v\right) \\
& =\left(f_{n}, v\right), \quad \forall(v, q) \in X_{H, h}^{0}(\Omega) \times M_{H, h}^{0}(\Omega), \\
& u_{0}^{H, h}=u^{H, h}(0) .
\end{aligned}
$$


To analyze the above local algorithm, following the proof of the Lemma 3.2 in [12] and [33], we can get following lemma.

Lemma 3.1. Suppose $D \subset \subset \Omega_{0} \subset \Omega$ and Assumptions (B1)-(B4) hold. Then $\left(w_{n}^{h}, r_{n}^{h}\right) \in X_{h}(\Omega) \times$ $M_{h}(\Omega),(n=1,2, \cdots, N)$, satisfying

$$
\begin{aligned}
& \frac{1}{\tau}\left(w_{n}^{h}-w_{n-1}^{h}, v\right)+B\left(\left(w_{n}^{h}, r_{n}^{h}\right) ;(v, q)\right)+N\left(b, w_{n}^{h}, v\right) \\
& =\left(f_{n}, v\right), \quad \forall(v, q) \in X_{0}^{h}\left(\Omega_{0}\right) \times M_{0}^{h}\left(\Omega_{0}\right), \\
& w_{0}^{h}=w^{h}(0),
\end{aligned}
$$

has the following local estimate:

$$
\tau^{1 / 2}\left\|w_{n}^{h}\right\|_{1, D} \leq c\left(\left(1+\tau^{1 / 2}\right)\left\|w_{n}^{h}\right\|_{0, \Omega_{0}}+\tau^{1 / 2}\left\|f_{n}\right\|_{-1, \Omega_{0}}+\left\|w_{n-1}^{h}\right\|_{0, \Omega_{0}}\right) .
$$

Theorem 3.1. Assume that the conditions of Theorem 2.4 and Assumptions (B1)-(B4) hold. $\left(u_{n}^{h}, p_{n}^{h}\right)$ is the solution of (2.7) and the solution $\left(u_{n}^{H, h}, p_{n}^{H, h}\right)$ is obtained from (3.1). Then for $D \subset \subset \Omega_{0}$ and $n=1,2, \cdots, N$, there holds

$$
\left\|u_{n}^{h}-u_{n}^{H, h}\right\|_{1, D}+\left\|p_{n}^{h}-p_{n}^{H, h}\right\|_{0, D} \leq c\left(1+\tau^{-1}\right)\left(\tau+H^{s+1}\right), \quad s=1,2 .
$$

Consequently,

$$
\left\|u\left(t_{n}\right)-u_{n}^{H, h}\right\|_{1, D}+\left\|p\left(t_{n}\right)-p_{n}^{H, h}\right\|_{0, D} \leq c\left(1+\tau^{-1}\right)\left(\tau+h^{s}+H^{s+1}\right), \quad s=1,2 .
$$

Proof. Thanks to the assumption on the auxiliary grid $T^{h}(\Omega)$ that coincides with $T_{H}^{h}(\Omega)$ on $\Omega_{0}$, we derive from (2.7) and Algorithm 0 that

$$
\begin{aligned}
& \frac{1}{\tau}\left(\left(u_{n}^{h}-u_{n}^{H, h}\right)-\left(u_{n-1}^{h}-u_{n-1}^{H, h}\right), v\right)+B\left(\left(u_{n}^{h}-u_{n}^{H, h}, p_{n}^{h}-p_{n}^{H, h}\right) ;(v, q)\right) \\
& \quad+N\left(b, u_{n}^{h}-u_{n}^{H, h}, v\right)=0, \quad \forall(v, q) \in X_{0}^{h}\left(\Omega_{0}\right) \times M_{0}^{h}\left(\Omega_{0}\right) \\
& u_{0}^{h}-u_{0}^{H, h}=0 .
\end{aligned}
$$

By applying Lemma 3.1, Theorem 2.4 and (2.3b), we get

$$
\begin{array}{ll}
\tau^{1 / 2}\left\|u_{n}^{h}-u_{n}^{H, h}\right\|_{1, D} & \\
\leq c\left(\left(1+\tau^{1 / 2}\right)\left\|u_{n}^{h}-u_{n}^{H, h}\right\|_{0, \Omega_{0}}+\left\|u_{n-1}^{h}-u_{n-1}^{H, h}\right\|_{0, \Omega_{0}}\right. & \\
\leq c\left(\left(1+\tau^{1 / 2}\right)\left\|u\left(t_{n}\right)-u_{n}^{h}\right\|_{0, \Omega_{0}}+\left(1+\tau^{1 / 2}\right)\left\|u\left(t_{n}\right)-u_{n}^{H, h}\right\|_{0, \Omega_{0}}\right. & \\
\quad \quad+\left\|u\left(t_{n-1}\right)-u_{n-1}^{h}\right\|_{0, \Omega_{0}}+\left\|u\left(t_{n-1}\right)-u_{n-1}^{H, h}\right\|_{0, \Omega_{0}} & \\
\leq c\left(1+\tau^{1 / 2}\right)\left(\tau+H^{s+1}\right), & s=1,2, \\
\left\|u_{n}^{h}-u_{n}^{H, h}\right\|_{1, D} \leq c\left(1+\tau^{-1 / 2}\right)\left(\tau+H^{s+1}\right) \leq c\left(1+\tau^{-1}\right)\left(\tau+H^{s+1}\right), & s=1,2 .
\end{array}
$$


Combining the triangle inequality with (2.9a), we see

$$
\left\|u\left(t_{n}\right)-u_{n}^{H, h}\right\|_{1, D} \leq c\left(1+\tau^{-1}\right)\left(\tau+h^{s}+H^{s+1}\right), \quad s=1,2 .
$$

On the other hand, from the inf-sup condition and (3.4), we get

$$
\begin{aligned}
\left\|p_{n}^{h}-p_{n}^{H, h}\right\|_{0, D} & \leq c \tau^{-1}\left(\left\|u_{n}^{h}-u_{n}^{H, h}\right\|_{0, D}+\left\|u_{n-1}^{h}-u_{n-1}^{H, h}\right\|_{0, D}\right)+c\left|u_{n}^{h}-u_{n}^{H, h}\right|_{1, D} \\
& \leq c \tau^{-1}\left(\tau+H^{s+1}\right)+c\left(1+\tau^{-1 / 2}\right)\left(\tau+H^{s+1}\right) \\
& \leq c\left(1+\tau^{-1}\right)\left(\tau+H^{s+1}\right), \quad s=1,2 .
\end{aligned}
$$

Combining it with (2.9b), we get (3.3).

\section{Parallel finite element algorithm}

Given an initial coarse grid $T^{H}(\Omega)$, let us first divide $\Omega$ into a number of disjoint subdomains $D_{1}, D_{2}, \cdots, D_{J}$, then enlarge each $D_{j}$ to obtain $\Omega_{j}$ such that $D_{j} \subset \subset \Omega_{j} \subset \Omega(j=$ $1,2, \cdots, J)$. For each $\Omega_{j}$, we use some local refinement and adaptive processes to obtain a global composite mesh $T_{j}^{H, h}(\Omega)(j=1,2, \cdots, J)$. We note that each $T_{j}^{H, h}(\Omega)$ has a substantially finer mesh inside $\Omega_{j}$ and a much coarse mesh away from $\Omega_{j}$. All of these $T_{j}^{H, h}(\Omega)$ compose a fully overlapping domain decomposition of $\Omega$. It is noted that all of $T_{j}^{H, h}(\Omega)$ are different triangulations of $\Omega$ and they can be very arbitrary; but for simplicity of exposition, we assume that each $T_{j}^{H, h}(\Omega)$ has the same size $h$ in $\Omega_{j}$ and has the size $H$ away from $\Omega_{j}$. Let $X_{H, h, j}^{0}(\Omega) \subset X, M_{H, h, j}^{0}(\Omega) \subset M$ be the corresponding mixed finite element spaces associated with the composite mesh $T_{j}^{H, h}(\Omega)$. Our parallel finite element algorithm reads as follows.

Algorithm 4.1 (Parallel finite element algorithm). For $n=1,2, \cdots, N$,

1. Find $\left(u_{j, n}^{H, h}, p_{j, h}^{H, h}\right) \in X_{H, h, j}^{0}(\Omega) \times M_{H, h, j}^{0}(\Omega),(j=1,2, \cdots, J)$ in parallel, such that

$$
\begin{aligned}
& \frac{1}{\tau}\left(u_{j, n}^{H, h}-u_{j, n-1}^{H, h}, v\right)+B\left(\left(u_{j, n}^{H, h}, p_{j, n}^{H, h}\right) ;(v, q)\right)+N\left(b, u_{j, n}^{H, h}, v\right) \\
& =\left(f_{n}, v\right), \quad \forall(v, q) \in X_{H, h, j}^{0}(\Omega) \times M_{H, h, j}^{0}(\Omega), \\
& u_{j, 0}^{H, h}=P_{H, h, j} u_{0} .
\end{aligned}
$$

2. Set $\left(\bar{u}_{n}^{h}, \bar{p}_{n}^{h}\right)=\left(u_{j, n}^{H, h}, p_{j, n}^{H, h}\right)$ in $D_{j},(j=1,2, \cdots, J)$. 
Defining piecewise norms:

$$
\begin{aligned}
& \|\| u\left(t_{n}\right)-\bar{u}_{n}^{h}|\||_{1, \Omega}=\left(\sum_{j=1}^{J}\left\|u\left(t_{n}\right)-\bar{u}_{n}^{h}\right\|_{1, D_{j}}^{2}\right)^{1 / 2}, \\
& \left.\left\|\left|p\left(t_{n}\right)-\bar{p}_{n}^{h}\right|\right\|\right|_{0, \Omega}=\left(\sum_{j=1}^{J}\left\|p\left(t_{n}\right)-\bar{p}_{n}^{h}\right\|_{0, D_{j}}^{2}\right)^{1 / 2},
\end{aligned}
$$

and from Theorem 3.1, we have the following result.

Theorem 4.1. Assume that the conditions of Theorem 3.1 hold. The solution $\left(\bar{u}_{n}^{h}, \bar{p}_{n}^{h}\right)$ obtained from Algorithm 4.1 satisfies for $n=1,2, \cdots, N$, that

$$
\left.\left\|\left|u\left(t_{n}\right)-\bar{u}_{n}^{h}\left\|\left.\right|_{1, \Omega}+\right\|\right| p\left(t_{n}\right)-\bar{p}_{n}^{h}\right\|\right|_{0, \Omega} \leq c\left(1+\tau^{-1}\right)\left(\tau+h^{s}+H^{s+1}\right), \quad s=1,2 .
$$

Proof. From Theorem 3.1, we obtain

$$
\left\|u\left(t_{n}\right)-\bar{u}_{n}^{h}\right\|_{1, D_{j}}+\left\|p\left(t_{n}\right)-\bar{p}_{n}^{h}\right\|_{0, D_{j}} \leq c\left(1+\tau^{-1}\right)\left(\tau+h^{s}+H^{s+1}\right), \quad s=1,2, \quad j=1,2, \cdots, J .
$$

By a collection of subdomains $D_{j},(j=1,2, \cdots, J)$, we can get the result (4.2).

Remark 4.1. From Theorem 4.1, we see that the scaling between the coarse and fine mesh sizes satisfies $H^{s+1}=\mathcal{O}\left(h^{s}\right)$. For instance, $H=\mathcal{O}\left(h^{1 / 2}\right)$ when $s=1$, while $H=\mathcal{O}\left(h^{2 / 3}\right)$ when $s=2$.

\section{Numerical results}

In this section, we shall present three kinds of numerical results to verify the efficiency of our parallel finite element algorithm. The first one is a problem with known analytic solution problem, the second one is the lid-driven cavity flow problem and the third one is the flow around a circular cylinder. In our experiments, the computer CPU is Inter dual-core Core i5 3337U 1.80GHz, 4GB memory. The algorithm is implemented by using the public domain software FreeFem++ [52] and the finite element discretization uses Taylor-Hood elements.

\subsection{Analytic solution}

In this test example, $\Omega$ is the unit square $[0,1] \times[0,1] \subset R^{2}$. We set $f$ and the boundary conditions such that the exact solution of the unsteady Oseen problem is given by

$$
\begin{aligned}
& u_{1}=x^{2}(x-1)^{2} y(y-1)(2 y-1) e^{-t}, \\
& u_{2}=-y^{2}(y-1)^{2} x(x-1)(2 x-1) e^{-t}, \\
& p=(2 x-1)(2 y-1) e^{-t}, \\
& b_{1}=\sin (x) \sin (y) \\
& b_{2}=\cos (x) \cos (y) .
\end{aligned}
$$


Table 1: Errors of the approximate solutions: $v=1, \tau=0.001, T=0.01$.

\begin{tabular}{||c|ccccccc||}
\hline Method & $h$ & $H$ & $C P U(s)$ & $\frac{\left\|\nabla\left(u(T)-\bar{u}_{N}^{h}\right)\right\| \|_{0, \Omega}}{\|\nabla u(T)\|_{0, \Omega}}$ & $\frac{\left\|p(T)-\bar{p}_{N}^{h} \mid\right\|_{0, \Omega}}{\|p(T)\|_{0, \Omega}}$ & $u_{H^{1}}-$ rate & $p_{L^{2}}-$ rate \\
\hline \multirow{4}{*}{ Algorithm 4.1 } & $1 / 27$ & $1 / 18$ & 4.406 & 0.00705274 & 0.00174809 & - & - \\
& $1 / 64$ & $1 / 32$ & 21.094 & 0.00122302 & 0.000335677 & 2.04172 & 1.92357 \\
& $1 / 125$ & $1 / 50$ & 80.438 & 0.000285369 & $8.65976 \mathrm{e}-005$ & 2.18886 & 2.03886 \\
& $1 / 216$ & $1 / 72$ & 247.126 & $9.56311 \mathrm{e}-005$ & $2.9647 \mathrm{e}-005$ & 2.01711 & 1.97803 \\
\hline \multirow{5}{*}{ Standard FEM } & $1 / 27$ & - & 5.752 & 0.00403435 & 0.00106256 & - & - \\
& $1 / 64$ & - & 33.172 & 0.000727341 & 0.000191011 & 1.99665 & 2.00001 \\
& $1 / 125$ & - & 139.204 & 0.000192714 & $5.05757 \mathrm{e}-005$ & 1.99899 & 2 \\
& $1 / 216$ & - & 460.760 & $6.52993 \mathrm{e}-005$ & $1.71082 \mathrm{e}-005$ & 1.99688 & 1.99997 \\
\hline
\end{tabular}

Table 2: Errors of the approximate solutions: $v=1, \tau=0.0001, T=0.01$.

\begin{tabular}{|c|c|c|c|c|c|c|c|}
\hline Method & $h$ & $H$ & $C P U(s)$ & $\frac{\left\|\nabla\left(u(T)-\bar{u}_{N}^{h}\right) \mid\right\|_{0, \Omega}}{\|\nabla u(T)\|_{0, \Omega}}$ & $\frac{\left\|\left|p(T)-\bar{p}_{N}^{h}\right|\right\|_{0, \Omega}}{\|p(T)\|_{0, \Omega}}$ & $u_{H^{1}}-$ rate & $p_{L^{2}}-$ rate \\
\hline \multirow{4}{*}{ Algorithm 4.1} & $1 / 27$ & $1 / 18$ & 45.813 & 0.00705275 & 0.00174809 & - & - \\
\hline & $1 / 64$ & $1 / 32$ & 214.188 & 0.00122314 & 0.000335711 & 2.04172 & 1.92357 \\
\hline & $1 / 125$ & $1 / 50$ & 811.975 & 0.000288953 & $8.6338 \mathrm{e}-005$ & 2.17052 & 2.04365 \\
\hline & $1 / 216$ & $1 / 72$ & 2395.48 & $9.56663 \mathrm{e}-005$ & $2.96548 \mathrm{e}-005$ & 2.03943 & 1.97224 \\
\hline \multirow{4}{*}{ Standard FEM } & $1 / 27$ & - & 56.798 & 0.00403434 & 0.00106256 & - & - \\
\hline & $1 / 64$ & - & 329.269 & 0.000727403 & 0.00019103 & 1.99667 & 2.00001 \\
\hline & $1 / 125$ & - & 1385.17 & 0.000192714 & $5.05858 \mathrm{e}-005$ & 1.99927 & 2 \\
\hline & $1 / 216$ & - & 4597.07 & $6.52039 \mathrm{e}-005$ & $1.71131 \mathrm{e}-005$ & 1.99974 & 2 \\
\hline
\end{tabular}

To test the asymptotical errors provided by our parallel finite element algorithm, we divide $\Omega=[0,1] \times[0,1]$ into four disjoint subdomains

$$
\begin{array}{ll}
D_{1}=(0,1 / 2) \times(0,1 / 2), & D_{2}=(1 / 2,1) \times(0,1 / 2), \\
D_{3}=(1 / 2,1) \times(1 / 2,1), & D_{4}=(0,1 / 2) \times(1 / 2,1),
\end{array}
$$

then extend each $D_{j},(j=1,2,3,4)$ outside with size $h$ to construct $\Omega_{j}$. We set $\nu=1, T=0.01$, and compute the finite element solutions on the composite meshes $T_{j}^{H, h}(\Omega),(j=1,2,3,4)$ independently by using Algorithm 4.1 and the standard one-level finite element method (Standard FEM) with $\tau=0.001,0.0001$, where the fine meshes of sizes $h=n^{-3},(n=3,4,5,6)$ and corresponding coarse meshes of size $H$ satisfying $H=0.5 h^{2 / 3}$. The numerical results are listed in Tables 1 and 2, respectively. In which, the CPU time is the maximum of the CPU time taken by the algorithm over the four subdomain, which includes the meshes generating time, the solving time of the problems and the error computing time. The convergence rates with respect to the mesh paramater $h$ are computed by the formula $\frac{\log \left(E_{i} / E_{i+1}\right)}{\log \left(h_{i} / h_{i+1}\right)}$, where $E_{i}$ and $E_{i+1}$ are the relative errors corresponding to the fine meshes of sizes $h_{i}$ and $h_{i+1}$, respectively.

From Tables 1 and 2, we can see that our parallel algorithm is highly efficient and can yield an approximate solution with an accuracy comparable to that of the standard onelevel finite element solution. However, our parallel algorithm saves a large amount of 


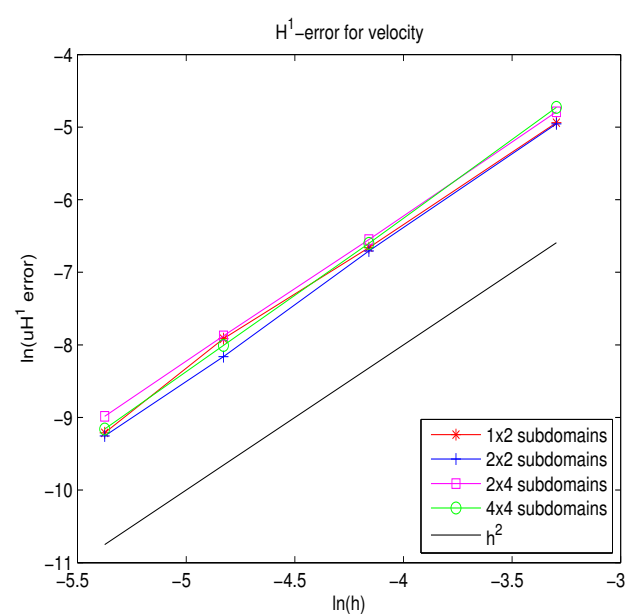

(a) velocity

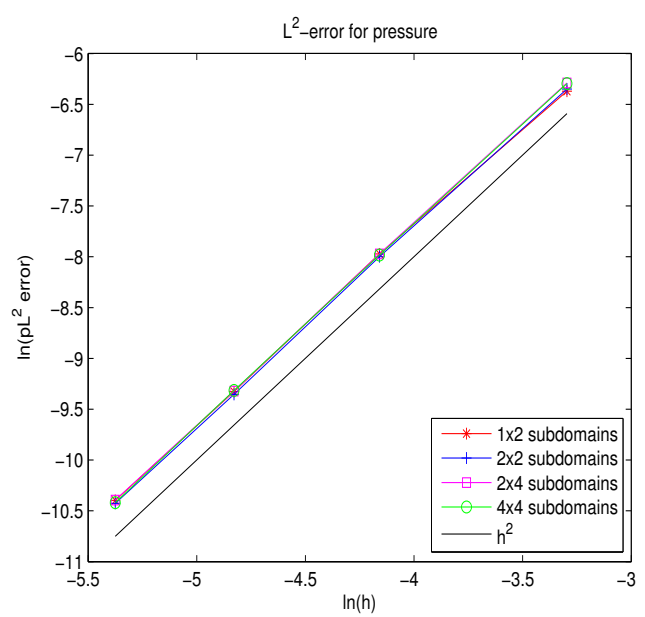

(b) pressure

Figure 2: The rates of convergence with different numbers of subregions.

computational time compared with the standard one-level finite element method. Moreover, comparing Tables 1 and 2, we also can observe that there is not much difference in rates of convergence and accuracy of the approximate solutions of velocity and pressure with different time step sizes.

Secondly, in order to compare the effects of number of subregions on the approximate solution of the parallel finite element algorithm, we divide the solution domain $\Omega$ into $1 \times 2,2 \times 2,2 \times 4$ and $4 \times 4$ subregions of equal size and then calculate the finite element approximation solutions applying Algorithms 4.1 with $v=1, h=1 / 216$ and $H=1 / 72$. Fig. 2 plots the rates of convergence of the approximate solutions of velocity and pressure with different numbers of subregions. We can observe that there is not much difference in rates of convergence and accuracy of the approximate solutions of velocity and pressure with different numbers of subregions.

\subsection{Lid-driven cavity flow}

In this test case, we consider the $2 D$ incompressible lid-driven cavity flow problem defined on a unit square. The external body force is set as zero. The boundary conditions are shown in Fig. 3 . We set the grid size $h=1 / 216, H=1 / 72$, viscosity coefficient $v=1$, time step $\tau=0.01, T=1, b_{1}=1, b_{2}=1$. Fig. 4 depicts the computed streamlines by Algorithm 4.1 with $J=4, J=8, J=16$ and the standard one-level finite element method, respectively. Note that the horizontal and vertical lines in Fig. 4 are the artificial boundaries of non-overlapping subdomains. This test case further illustrated the effectiveness of our parallel finite element algorithm. 


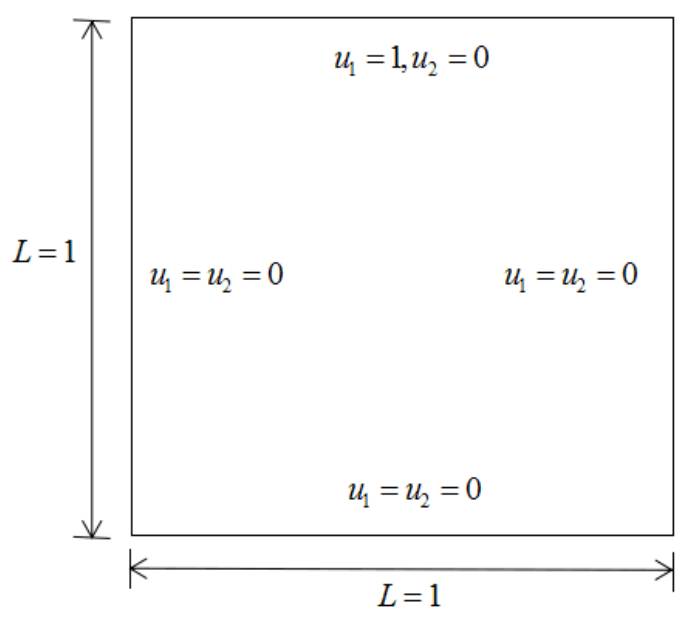

Figure 3: The lid-driven cavity flow problem.

\subsection{Flow around a circular cylinder}

To show that our proposed method works well for practical problem, we give a numerical test for the benchmark problem of $2 D$ channel flow around a circular cylinder defined on $\Omega=[0,6] \times[0,1]$, where a circle of radius 0.15 centers at $(x, y)=(1,0.5)$. The unsteady inflow velocity profiles are given by

$$
u_{1}(x, y, t)=6 y(1-y), \quad u_{2}(x, y, t)=0
$$

and the boundary condition of the outlet is set as

$$
-p I+\frac{\partial u}{\partial n}=0
$$

while no-slip boundary conditions are imposed on the other boundaries of the channel as well as the surface of the cylinder. The body force is set as $f=0$. The Taylor-Hood elements are used for spacial discretization and Euler scheme is used. We set time step size $\tau=0.01$ with final time $T=2$, the kinematic viscosity $v=1$ and then compute the approximate solutions by our parallel finite element algorithm with the meshes size $h=1 / 8$, $H=1 / 4$ and the standard one-level finite element method with a mesh size $h=1 / 8$, where the solution domain is decompose into three disjoint subdomain. Fig. 5 describes the contours of velocity and pressure computed by our parallel algorithm and the standard one-level finite element method, from which we can observe that the proposed algorithm in this paper predicts the flow structures well and demonstrates the effectiveness and feasibility of the algorithm. 


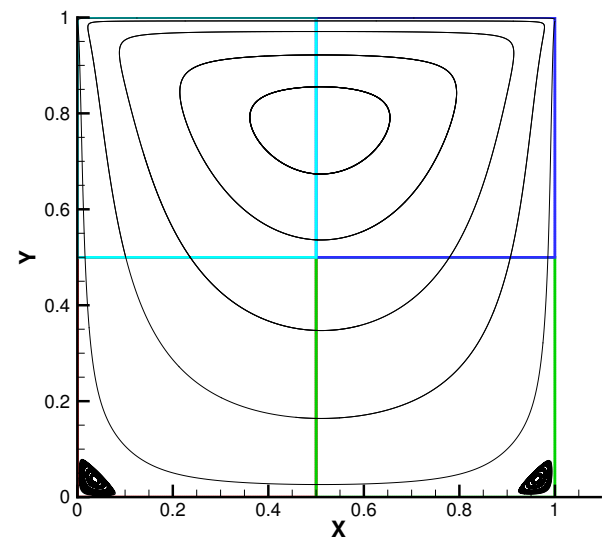

(a) Algorithm 4.1 with $J=4$

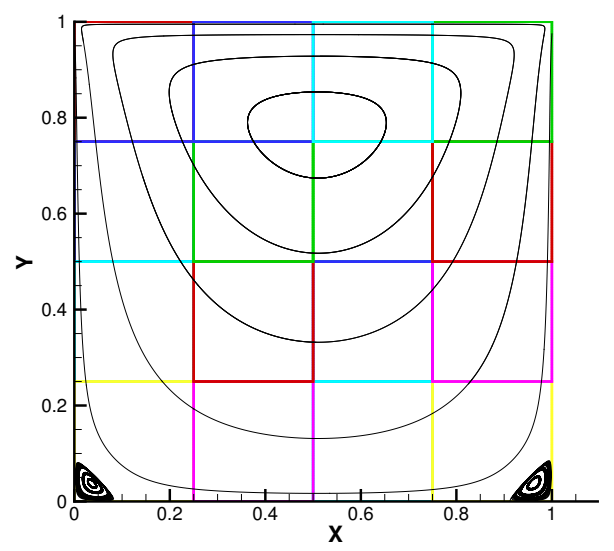

(c) Algorithm 4.1 with $J=16$

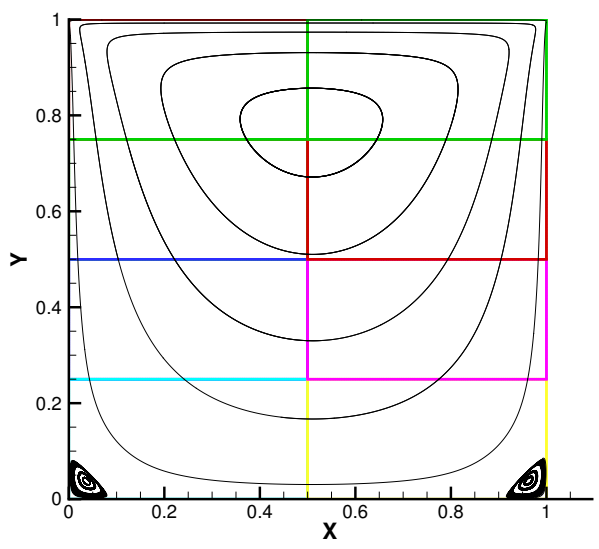

(b) Algorithm 4.1 with $J=8$

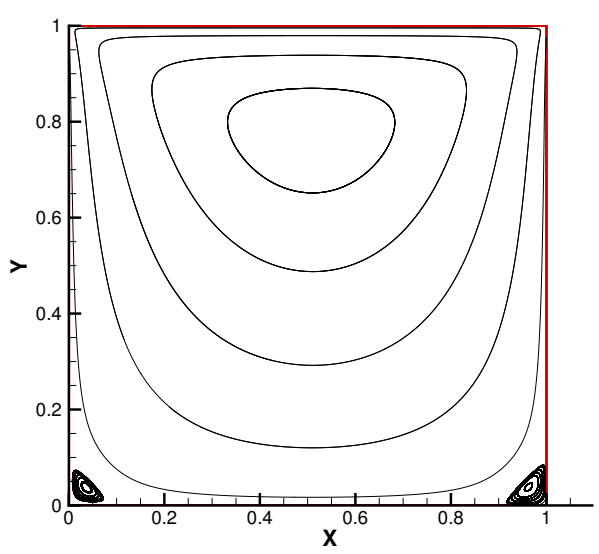

(d) Standard FEM

Figure 4: Streamlines of the computed solutions from Algorithm 4.1 and Standard FEM for the lid-driven cavity flow.

\section{Conclusions}

Based on the fully overlapping domain decomposition technique, this paper proposes a parallel finite element algorithm for solving the unsteady Oseen equations. By selecting appropriate mesh sizes, our proposed algorithm can achieve the same convergence order as the standard one-level finite element method, while saving a large amount of computational time. Finally, the efficiency of the parallel finite element algorithm is verified by numerical experiments on a problem with known analytic solutions, the lid-driven cavity flow and the flow around a circular cylinder. 


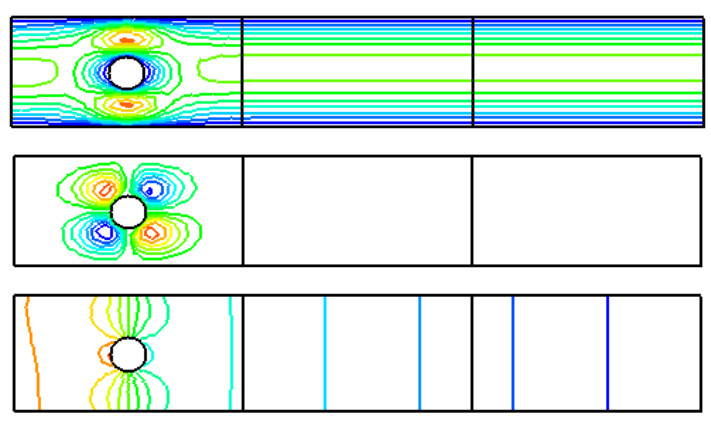

(a) Algorithm 4.1
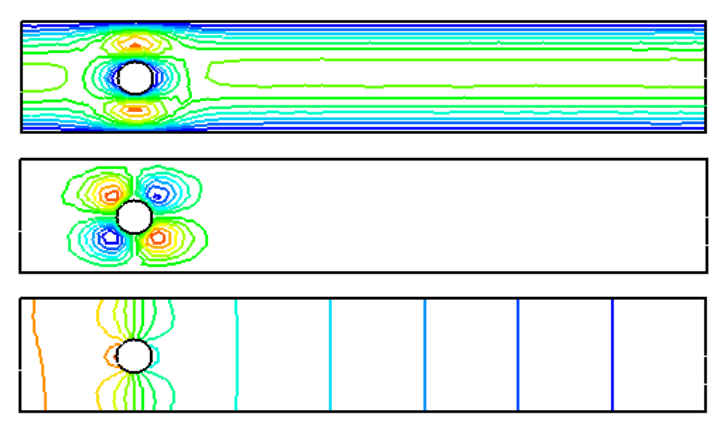

(b) Standard FEM

Figure 5: Contours of velocity and pressure by Algorithm 4.1 and Standard FEM for the flow around a circular cylinder.

\section{Acknowledgements}

The authors would like to express their deep gratitude to the anonymous reviewers for their valuable comments and suggestions, which led to an improvement of the paper.

This work was supported by the Natural Science Foundation of China (No. 11361016), the Basic and Frontier Explore Program of Chongqing Municipality, China (No. cstc2018jcyjAX0305) and Funds for the Central Universities (No. XDJK2018B032).

\section{References}

[1] J. C. XU AND A. H. ZHOU, Local and parallel finite element algorithms based on two-grid discretizations, Math. Comput., 69(231) (2000), pp. 881-909.

[2] J. C. XU AND A. H. ZHOU, Local and parallel finite element algorithms based on two-grid discretizations for nonlinear problems, Adv. Comput. Math., 14(4) (2001), pp. 293-327.

[3] J. C. XU AND A. H. ZHOU, Local and parallel finite element algorithms for eigenvalue problems, Acta Math. Appl. Sinica, English Series, 18 (2002), pp. 185-200.

[4] L. SHEN, Parallel Adaptive Finite Element Algorithms for Electronic Structure Computing Based on Density Functional Theory, PhD Thesis, Academy of Mathematics and Systems Science, Chinese Acade my of Sciences, 2005.

[5] H. BI, J. HAN AND Y. YANG, Local and parallel finite element algorithms for the transmission eigenvalue problem, J. Sci. Comput., 78(1) (2019), pp. 351-375.

[6] Y. ZHANG, Y. HOU, L. SHAN AND X. DONG, Local and parallel finite element algorithm for stationary incompressible magnetohydro dynamics, Numer. Methods Partial Differential Equations, 33(5) (2017), pp. 1513-1539.

[7] Y. N. HE, J. C. XU, A. H. ZHOU AND J. LI, Local and parallel finite element algorithms for the Stokes problem, Numer. Math., 109(3) (2008), pp. 415-434. 
[8] Y. Q. SHANG, A parallel stabilized finite element method based on the lowest equal-order elements for incompressible flows, Computing, 102(2020), pp. 65-81.

[9] K. R. ZHOU AND Y. Q. SHANG, Local and parallel finite element algorithms for the Stokes equations with nonlinear slip boundary conditions, Int. J. Comput. Methods, 17(8)(2020), id:1950050.

[10] G. Z. DU AND L. Y. ZUO, Local and parallel finite element post-processing scheme for the Stokes problem, Comput. Math. Appl., 73(1)(2017), pp. 129-140.

[11] G. Z DU AND L. Y. ZUO, Local and parallel finite element methods for the coupled Stokes/Darcy model, Numer. Algor., DOI: 10.1007/s11075-020-01021-5.

[12] Y. N. HE, J. C. XU AND A. H. ZHOU, Local and parallel finite element algorithms for the NavierStokes problem, J. Comput. Math., 24(3) (2006), pp. 227-238.

[13] Y. Q. Shang AND Y. N. HE, Parallel iterative finite element algorithms based on full domain partition for the stationary Navier-Stokes equations, Appl. Numer. Math., 60 (2010), pp. 719 737.

[14] Y. Q. SHANG, A parallel two-level linearization method for incompressible flow problems, Appl. Math. Lett., 24 (2011), pp. 364-369.

[15] Y. Q. SHANG AND Y. N. HE, A parallel Oseen-linearized algorithm for the stationary NavierStokes equations, Comput. Methods Appl. Mech. Eng., 209-212 (2012), pp. 172-183.

[16] Y. Q. SHANG, A parallel two-level finite element variational multiscale method for the NavierStokes equations, Nonlinear Anal., 84 (2013), pp. 103-116.

[17] Y. Q. SHANG AND S. M. HUANG, A parallel subgrid stabilized finite element method based on two-grid discretization for simulation of 2D/3D steady incompressible flows, J. Sci. Comput., 60 (2014), pp. 564-583.

[18] Y. Q. SHANG AND J. QIN, Parallel finite element variational multiscale algorithms for incompressible flow at high Reynolds numbers, Appl. Numer. Math., 117 (2017), pp. 1-21.

[19] B. ZHeng AND Y. Q. SHANG, Parallel iterative stabilized finite element algorithms based on the lowest equal-order elements for the stationary Navier-Stokes equations, Appl. Math. Comput., 357 (2019), pp. 35-56.

[20] B. ZHENG AND Y. Q. SHANG, A parallel stabilized finite element variational multiscale method based on fully overlapping domain decomposition for the incompressible Navier-Stokes equations, Appl. Numer. Math., 159 (2021), pp. 138-158.

[21] Y. SHU, J. LI, JiAn AND C. ZHANG, A local and parallel Uzawa finite element method for the generalized Navier-Stokes equations, Appl. Math. Comput., 387(2020), id: 124671.

[22] J. P. YU, F. SHI AND H. B. ZHENG, Local and parallel finite element algorithms based on the partition of unity for the Stokes problem, SIAM J. Sci. Copmut., 36(5)(2014), pp. C547-C567.

[23] H. B. ZHENG, J. P. YU AND F. SHI, Local and parallel finite element algorithm based on the partition of unity for incompressible flows, J. Sci. Comput., 65(2)(2015), pp. 512-532.

[24] G. L. ZhanG, H. Y. SU AND X. L. FENG, A novel parallel two-step algorithm based on finite element discretization for the incompressible flow problem, Numer. Heat Transfer Part BFundamentals, 73(5) (2018), pp. 329-341.

[25] F. Y. MA, Y. C. MA AND W. F. WO, Local and parallel finite element algorithms based on twogrid discretization for steady Navier-Stokes equations, Appl. Math. Mech., English Edition, 28(1) (2007), pp. 27-35.

[26] Y. C. MA, Z. P. ZHANG AND C. F. REN, Local and parallel finite element algorithms based on twogrid discretization for the stream function form of Navier-Stokes equations, Appl. Math. Comput., 175 (2006), pp. 786-813.

[27] X. LiU, J. Li AND Z. X. ChEN, A weak Galerkin finite element method for the Oseen equations, Adv. Comput. Math., 42(6) (2016), pp. 1473-1490. 
[28] M. Winter, B. SchotT, A. MAssing AND W. A. WAll, A Nitsche cut finite element method for the Oseen problem with general Navier boundary conditions, Comput. Methods Appl. Mech. Eng., 330 (2018), pp. 220-252.

[29] A. Massing, B. Schott And W. A. WAll, A stabilized Nitsche cut finite element method for the Oseen problem, Comput. Methods Appl. Mech. Eng., 328 (2018), pp. 262-300.

[30] C. XU, D. Y. SHI AND X. LIAO, A new streamline diffusion finite element method for the generalized Oseen problem, Appl. Math. Mech., 39(2) (2018), pp. 1-14.

[31] X. Y. ZHAO, Y. R. HOU AND G. Z. DU, A parallel partition of unity method for the unsteady Stokes equations, Int. J. Numer. Methods Heat Fluid Flow, 27(9) (2017), pp. 2105-2114.

[32] G. Z. DU AND L. Y. ZUO, A parallel partition of unity method for the nonstationary Navier-Stokes equations, Int. J. Numer. Methods Heat Fluid Flow, 27(8)(2017), pp. 1675-1686.

[33] Y. Q. SHANG AND K. WANG, Local and parallel finite element algorithms based on two-grid discretizations for the transient Stokes equations, Numer. Algorithms, 54(2) (2010), pp. 195-218.

[34] R. ADAMS, Sobolev Spaces, Academaic Press Inc, New York, 1975.

[35] P. G. Ciarlet, The Finite Element Method for Elliptic Problems, North-Holland, Amsterdam, 1978.

[36] P. G. Ciarlet And J. L. Lions, Handbook of Numerical Analysis, vol. II, Finite Element Methods (Part I), Elsevier Science Publisher, Amsterdam, 1991.

[37] A. T. HILL AND E. SLI, Approximation of the global attractor for the incompressible Navier-Stokes equations, IMA J. Numer. Anal., 20(4) (2000), pp. 633-667.

[38] J. G. HEYWOOD AND R. RANNACHER, Finite element approximation of the nonstationary Navier-Stokes problem III: smoothing property and higher order error estimates for spatial discretization, SIAM J. Numer. Anal., 25(3) (1988), pp. 489-512.

[39] J. G. HEYWOOD AND R. RANNACHER, Finite element approximation of the nonstationary Navier-Stokes problem I: regularity of solutions and second-order error estimates for spatial discretization, SIAM J. Numer. Anal., 19(2) (1982), pp. 275-311.

[40] D. N. ARNOLD AND X. LIU, Local error estimates for finite element discretizations of the Stokes equations, RAIRO M2AN 29 (1995), pp. 367-389.

[41] V. Girault and P. A. Raviart, Finite Element Method for Navier-Stokes Equations: Theory and Algorithms, Springer-Verlag, Berlin, Heidelberg, 1987.

[42] A. H. SCHATZ AND L. B. WAHLBIN, Interior maximum norm estimates for finite element methods, Math. Comput., 31 (1977), pp. 414-442.

[43] A. H. SCHATZ AND L. B. WAHLBIN, Interior maximum-norm estimates for finite element methods, part II, Math. Comput., 64 (1995), pp. 907-928.

[44] L. B. WAHLBIN, Superconvergence in Galerkin Finite Element Methods, Lecture Notes in Mathematics, Springer, 1605 (1995).

[45] V. JOHN, Finite element methods for incompressible flow problems, Springer International Publishing, 2016.

[46] D. N. ARnold, M. Fortin AND F. BrezZI, A stable finite element method for the stokes equations, Calcolo, 21(4) (1984), pp. 337-344.

[47] M. ForTin, Calcul Numérique des Ecoulements Fluides de Bingham et des Fluides Newtoniens Incompressible par des Méthodes d' eléments Finis, Doctoral thesis, Université de Paris VI, 1972.

[48] P. HOOD AND C. TAYLOR, A numerical solution of the Navier-Stokes equations using the finite element technique, Comput. Fluids, 1 (1973), 73-100.

[49] M. CROUZEIX AND P.-A. RAVIART, Conforming and nonconforming finite element methods for solving the stationary Stokes equations, RAIRO Anal. Numer., 7(R-3) (1973), pp. 33-76. 
[50] L. MANSFIELD, Finite element subspaces with optimal rates of convergence for stationary Stokes problem, Rairo Anal. Numer. Numer. Anal., 16 (1982), pp. 49-66.

[51] Y. N. HE, The Euler implicit/explicit scheme for the 2D time-dependent Navier-Stokes equations with smooth or non-smooth initial data, Math. Comput., 77(264) (2008), pp. 2097-2124.

[52] F. HECHT, New development in Freefem++, J. Numer. Math., 20 (2012), pp. 251-265. 